\title{
44502 - AN UPDATE ON THE BETA BLOCKER META ANALYSIS: HEART RATE CONTROL REDUCES POST-OPERATIVE MI
}

\author{
Farhan Husain, Toronto General Hospital, University Health Network, Toronto, ON, \\ Canada; \\ W Scott Beattie, Toronto General Hospital, University Health Network; \\ Duminda Wijeysundera, Toronto General Hospital, University Health Network; \\ K Karkouti, Toronto General Hospital, University Health Network; \\ S McCluskey, Toronto General Hospital, University Health Network; \\ G Tait, PhD;
}

INTRODUCTION: To date, no meta-analyses assessing the efficacy of perioperative betablockade trials has considered the influence of heart rate control. A systematic review of all perioperative beta blockade studies prior to 2005 suggested that there was not enough evidence to support the universal perioperative use of beta-blockers. Since then 6 studies have been published, half of which show benefit $(1,2,3)$ while the other half cannot show such a correlation $(4,5,6)$. The purpose of this study is to update the systematic review with newer trials, published in the last year, and to consider the influence of tight heart rate control and agent half-life on the efficacy of perioperative beta blockade.

METHODS: For the purpose of this analysis the outcome of interest was in hospital myocardial infarction (MI) after non-cardiac surgery in all randomized trials evaluating perioperative betablockers. Relative Risk (RR) and 95\% confidence intervals (CI) were calculated using REVMAN 4.2 (Cochrane collaboration). Two subgroup analyses were carried out comparing the efficacy of short and long half-life beta-blockers and the degree of heart rate control. The measure of heart rate control was the upper $95 \%$ CI for postoperative heart rate (HR) in patients receiving beta-blockers. This index of heart rate control was plotted against RR for MI in each trial.

RESULTS: The results of the updated analysis are seen in figure 1. Patients receiving short acting beta-blockers were not protected against perioperative MI (RR 1.08; 95\% CI 0.74-1.49; $\mathrm{P}=0.69$ ), whereas longer acting and continuous infusion beta-blockers were (RR 0.20; 95\% CI 0.06-0.64; $\mathrm{P}=0.007)$. The longer acting agents were associated with less variability in heart rates as compared to the shorter acting agents.

DISCUSSION: This updated review of the RCT's assessing perioperative beta-blockade shows that both the duration of agent half-life and the heart rate control are important determinants of efficacy. Trials where longer acting beta-blockers were used or where beta-blockers achieved HR control showed a cardio protective effect.

REFERENCES: 1.J Vasc Surg 2005;41:602-9, 2. BMJ 2006;332:1482 3.Am Heart J 2006;152:983-90, 4.J Am Coll Cardiol 2006;48:964-9, 5.Circulation 2006;114:I344-I349, 6.Anesth Analg 1999;89:801 


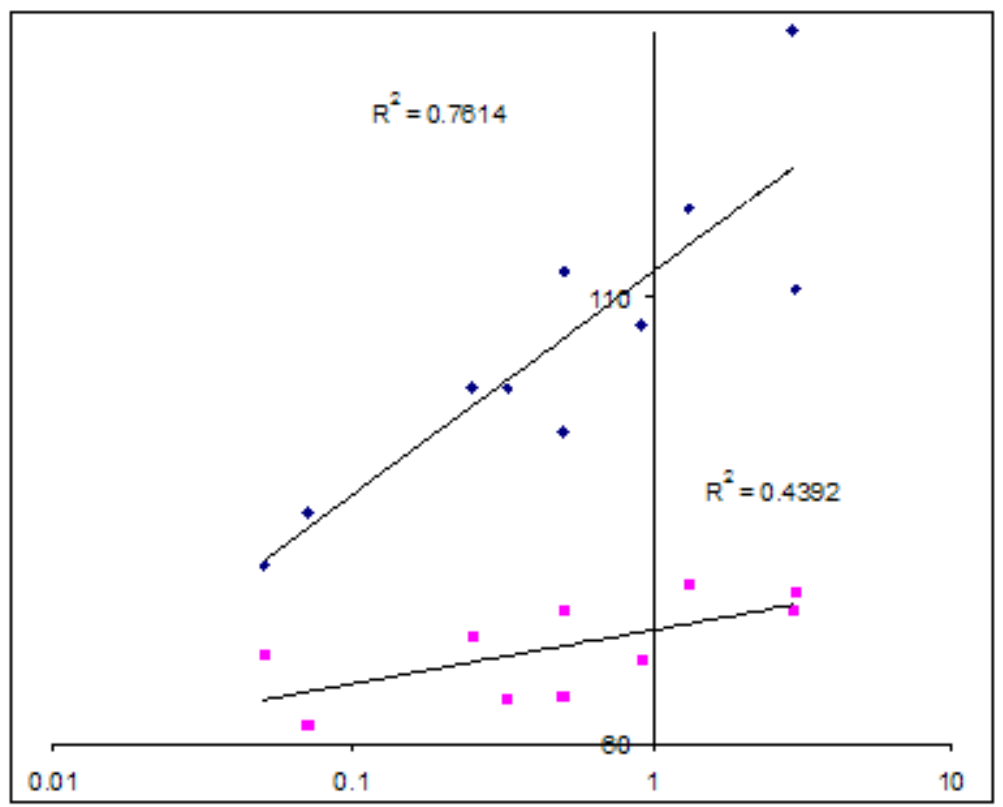

Figure 1 The effect of mean heart rates and maximal heart rates on the log odds ratio of a post operative MI. The squares represent the mean HR of a trial the diamonds represent the maximal HR recorded. There are 3 statistically significant relationships 1 ) increasing mean heart rate and increasing $R R$ of $M I r=67.2$ ) Increasing Maximal heart rates and increasing odds of a MI $r=0.87$ and 3 ) the difference between man and maximal $\mathrm{HR}$ and increasing $R R$ of an MI r $=0.81$ 